\title{
Use of frozen-thawed sperm for ICSI improves fertility outcome in men with azoospermia
}

\author{
๑Arzu Yurci ${ }^{1}$, @Nur Dokuzeylül Güngör ${ }^{2}$ \\ ${ }^{1}$ Memorial Kayseri Hospital, Department of Gynecology Obstetrics and Reproductive Medicine, IVF Center, Kayseri, Turkey \\ ${ }^{2}$ Medicalpark Göztepe Hospital, Department of Gynecology Obstetrics and Reproductive Medicine, IVF Clinic, İstanbul, Turkey
}

Cite this article as: Yurci A, Dokuzeylül Güngör N. Use of frozen-thawed sperm for ICSI improves fertility outcome in men with azoospermia. J Health Sci Med 2021; 4(3): 272-276.

\begin{abstract}
Aim: To compare the results of IVF/ICSI outcomes with fresh or frozen-thawed testicular sperm in patients who underwent mTESE with the diagnosis of azoospermia.

Material and Method: This retrospective cohort study was conducted on 260 infertile men who applied to the Memorila IVFCenter with the diagnosis of azoospermia between 2017 and 2020 and underwent surgical sperm extraction and ICSI using fresh or frozen testicular sperm. The patients were divided into two equal groups as indicated. Group $1(n=130)$ consisted of patients who underwent ICSI and subsequent embryo transfer using fresh testicular sperm. Group $2(n=130)$; consisted of patients who underwent ICSI by using frozen-thawed testicular sperm. Primary outcome was clinical pregnacy rates (CPR) and live birth rates (LBR). Patients in both groups who underwent ICSI/ET using frozen or fresh testicular sperm were compared in terms of clinical pregnancy and live birth rates. In addition, both groups were compared in terms of both demographic and other reproductive parameters.

Results: The total oocyte, M II oocyte and 2 PN embryo counts of the patients in the fresh sperm group were found to be significantly higher than the frozen sperm group $(\mathrm{p}<.000)$. In the fresh sperm the clinical pregnancy rate was detected in 25 cases $(19.8 \%)$, while no pregnancy was detected in 101 cases $(80.2 \%)$. In the frozen-thawed sperm group the clinical pregnancy was detected in 66 cases $(50.8 \%)$, whereas pregnancy was not detected in 64 cases $(49.2 \%)$. Clinical pregnancy rates were found to be significantly higher in patients who underwent ICSI/ET with frozen sperm compared to fresh sperm group (50.8\% vs $19.8 \%, \mathrm{p}<.000)$. Similarly, live birth rates were found to be significantly higher in the frozen sperm and ICSI/ET group compared to the fresh sperm group ( $3.2 \%$ vs $41.9 \%, \mathrm{p}<.00)$.

Conclusion: Use of frozen-thawed testicular sperm for ICSI/ET in men with azoospermia may provide better clinical pregnancy and live birt rates than ICSI cycles with fresh testicular sperm.
\end{abstract}

Keywords: Fresh sperm, frozen sperm, ICSI, azoospermia, fertility outcome

\section{INTRODUCTION}

Azoospermia is one of the most difficult causes of male infertility with a frequency of $10-15 \%$ among infertile men. Treatment chances of non-obstructive azoospermia forms are lower than obstructive azoopermia. With the development of surgical sperm retrieval methods such as microdissection testicular sperm extraction (microTESE), patients with azoospermia have begun to get the chance of pregnancy $(1,2)$. However, despite repeated MTESE procedures, the sperm retrieval rate does not exceed $60 \%$. For this reason, it is vital that the remaining sperm in mTESE be frozen after they are used for ICSI. Thanks to the frozen testicular sperm, the patient is protected from repetitive surgical interventions (3). However, whether the use of frozen testicular sperm for ICSI has an effect on reproductive parameters has been a matter of great curiosity. In a recent meta-analysis conducted by Yu et al (4), it was reported that the use of frozen testicular sperm or fresh testicular sperm did not have a significant effect on fertilization rate, implantation rate, and clinical pregnancy rate. However, Park et al (5). stated that the implantation rates and clinical pregnancy rates after ICSI with frozen sperm in azopermia cases were significantly higher than fresh sperm-ICSI cycles. For all these reasons, it is obvious that reproductive aoutcoms should be investigated with more comprehensive studies after ICSI using fresh or frozen sperm in azoospermia cases. 
In this context, this study was planned to investigate whether the perform of ICSI with fresh or frozen-thawed testicular sperms in patients with azoospermia improve reproductive otucome.

\section{MATERIAL AND METHOD}

This study was conducted in accordance with the Declaration of Helsinki. The study was carried out with the permission of the Research Ethics Committee of Memorial Kayseri Hospital (Date: 16/01/2021, Decision No: 8). Verbal informed consent was obtained from all participants at the time of enrollment.

This retrospective cohort study was conducted on infertile cases who applied to the Memorila IVF-Center with the diagnosis of azoospermia between 2017 and 2020 and underwent surgical sperm extraction and ICSI using fresh or frozen testicular sperm. We extracted all data of the study from electronic medical record system. Two semen analysis was performed in the male partners at least 3 weeks apart and upon 3 to 7 days of abstinence. Azoospermia was defined as the absence of sperm cells in the seminal fluid. The main criteria for inclusion in the study were (i) presence of sperm in MTESE, (ii) absence of karyotype anomaly in the male partner, and (iii) azoospermia patient with frozen testicular sperm. Participants with karyotype anomalies were not included in the study. Similarly, cases with no sperm in TESE were excluded from the study. The total number of patients meeting these criteria was determined to be 260 . All patients with a diagnosis of azoospermia who underwent mTESE and had sperm were included in the study. While 115 of 260 azoospermia cases were obstructive azoospermia, the remaining 145 cases were diagnosed as non onbstructive azoospermia. After some of the sperm obtained by testicular sperm extraction were used in ICSI, the remaining sperm were frozen. In some cases, there was not enough sperm left to freeze after ICSI.

The patients were divided into two equal groups as indicated. Group $1(n=130)$ consisted of patients who underwent ICSI and subsequent embryo transfer using fresh testicular sperm. Group $2(n=130)$; consisted of patients who underwent ICSI followed by ET using frozen testicular sperm. Most of the cases in Group 2 consisted of azoospermia cases who had previously undergone ICSI/ET with fresh testicular sperm but could not achieve live birth or presented to have a second baby. ICSI was repeated using frozen sperm in 85 cases who did not conceive with fresh sperm. While embryo transfer was possible in 126 of 130 cases in Group 1, transfer could not be made in 4 cases because there was no fertilization. In all 130 cases in Group 2, it was possible to perform ET after ICSI using frozen sperm. On the 3rd day and a single embryo transfer was performed to the cases in both groups. 130 couple with azoospermia who underwent ICSI using fresh sperm and 130 cases who underwent ICSI using frozen sperm were compared in terms of reproductive parametrers, clinical pregnancy rates, and live birth rates. Standard antagonist protocol was applied to both groups of participants. Controlled ovarian stimulation was performed with gonadotropin dose determined according to the patients' age, clinical findings and BMI evaluation.

Primary outcome was clinical pregnacy rates (CPR) and live birth rates (LBR). Patients in both groups who underwent ICSI/ET using frozen or fresh testicular sperm were compared in terms of clinical pregnancy and live birth rates. In addition, both groups were compared in terms of both demographic and other reproductive parameters. Clinical pregnancy rate defined as evidence of a gestational sac, confirmed by ultrasound examination. Live birth rate defined as delivery of a live fetus after 24 completed weeks of gestational age.

\section{Statistical Analysis}

The sample size was calculated with the GPower 3.1 (http://www.gpower.hhu.de/) program. The total mean of two groups compared based on the Mann-Whitney U test with the effect size of $40 \%$, power of $90 \%$ and 0.05 type 1 error, was found to be at least 113 patients. The results of Kolmogorov test show that not all quantitative variables have a normal distribution. Mann-Whitney $U$ test is used to examine the relationship between quantitative variables in the two Groups. Chi-square test and Fisher's exact test are used to examine qualitative variables. p-value $\leq 0.05$ was considered significant. Statistical Package for Social Sciences (SPSS) version 26.0 (SPSS Inc., Chicago, IL, USA) was used to perform data analysis.

\section{RESULTS}

While the minimum age of participants was 20 the maximum age was 43 (Table 1). The mean age of the all participants was $29.7( \pm 5.2)$. While the minimum infertility duration is one year and the maximum infertility duration is 20 years. The mean BMI was found $26.4( \pm 2.3)$. While 91 out of all participants had clinical pregnancy $(35.5 \%)$ the remaining 165 participants (64.5\%) had no clinical pregnancy. While 58 out of 256 participants (22.3\%) had live birth the remaining 198 participants (77.3\%) had no live birth. While $126(49.2 \%)$ out of 256 participants used ICSI with fresh sperm the remaining 130 subjects used ICSI with frozen sperm (50.8\%). Eighty-five patients who underwent ICSI with frozen sperm consisted of those who did not conceive after ICSI/ET with fresh sperm. When the patients were divided into two groups and analyzed, the mean age and 
duration of infertility of the frozen sperm group were significantly higher than that of the fresh sperm group (Table 2). The total oocyte, M II oocyte and $2 \mathrm{PN}$ embryo counts of the patients in the fresh sperm group were found to be significantly higher than the frozen sperm group $(\mathrm{p}<.000)$.

\begin{tabular}{|c|c|c|c|c|c|}
\hline Variable & $\mathbf{N}$ & Min. & Max. & Mean & SD \\
\hline Age (yrs) & 256 & 20 & 43 & 29.7 & 5.2 \\
\hline $\begin{array}{l}\text { Infertility } \\
\text { duration }\end{array}$ & 256 & 1 & 20 & 6.9 & 3.9 \\
\hline BMI & 256 & 23 & 32 & 26.4 & 2.3 \\
\hline Day 2 estradiol & 256 & 6 & 604.4 & 43.06 & 48.1 \\
\hline $\begin{array}{l}\text { Day } 2 \\
\text { progesterone }\end{array}$ & 256 & 0.01 & 0.7 & 0.2 & 0.17 \\
\hline $\begin{array}{l}\text { Total rFSH } \\
\text { dose }\end{array}$ & 256 & 800 & 5250 & 2278.1 & 906.5 \\
\hline $\begin{array}{l}\text { E2 on the day } \\
\text { of hCG }\end{array}$ & 256 & 0 & 9300 & 3149.3 & 1397.1 \\
\hline $\begin{array}{l}\text { Progeterone on } \\
\text { the day of hCG }\end{array}$ & 256 & 0 & 3.5 & 1.2 & 0.6 \\
\hline Total oocyte & 256 & 10 & 66 & 21.5 & 7.6 \\
\hline M II oocyte & 256 & 8 & 55 & 16.8 & 6.1 \\
\hline $2 \mathrm{PN}$ & 256 & 4 & 45 & 12.03 & 5.5 \\
\hline \multicolumn{2}{|c|}{ Clinical pregnancy } & \multicolumn{2}{|c|}{ Total } & \multicolumn{2}{|c|}{$\%$} \\
\hline \multicolumn{2}{|l|}{ Yes } & \multicolumn{2}{|c|}{91} & \multicolumn{2}{|c|}{35.5} \\
\hline No & & \multicolumn{2}{|c|}{165} & \multicolumn{2}{|c|}{64.5} \\
\hline \multicolumn{6}{|l|}{ Live birth } \\
\hline Yes & & \multicolumn{2}{|c|}{58} & \multicolumn{2}{|c|}{22.3} \\
\hline No & & \multicolumn{2}{|c|}{198} & \multicolumn{2}{|c|}{77.3} \\
\hline \multicolumn{6}{|l|}{ Groups } \\
\hline Fresh & & \multicolumn{2}{|c|}{126} & \multicolumn{2}{|c|}{49.2} \\
\hline Thaw & & \multicolumn{2}{|c|}{130} & \multicolumn{2}{|c|}{50.8} \\
\hline
\end{tabular}

Table 2. Investigating the significant relationship between groups and variables using Mann-Whitney U test

\begin{tabular}{|c|c|c|c|c|}
\hline \multirow[b]{2}{*}{ Variable } & \multicolumn{2}{|c|}{ Groups } & \multirow[b]{2}{*}{$\mathrm{Z}$} & \multirow[b]{2}{*}{$\begin{array}{c}P \\
\text { value }\end{array}$} \\
\hline & $\begin{array}{l}\text { ICSI with } \\
\text { fresh sperm } \\
\text { Mean (SD) }\end{array}$ & $\begin{array}{l}\text { ICSI with } \\
\text { frozen sperm } \\
\text { Mean (SD) }\end{array}$ & & \\
\hline Age (yrs) & $29(5.1)$ & $30.4(5.1)$ & -2.23 & 0.02 \\
\hline $\begin{array}{l}\text { Infertility } \\
\text { duration }\end{array}$ & $6.3(3.9)$ & $7.6(3.8)$ & -2.96 & 0.003 \\
\hline BMI & $26.4(2.3)$ & $26.4(2.3)$ & -0.01 & 0.9 \\
\hline $\begin{array}{l}\text { Day } 2 \\
\text { estradiol }\end{array}$ & $43.6(52.01)$ & $42.5(43.2)$ & -0.09 & 0.9 \\
\hline $\begin{array}{l}\text { Day } 2 \\
\text { progesterone }\end{array}$ & $0.2(0.1)$ & $0.2(0.1)$ & -0.4 & 0.6 \\
\hline $\begin{array}{l}\text { Total rFSH } \\
\text { dose }\end{array}$ & $\begin{array}{l}2154.9 \\
(919.5)\end{array}$ & $\begin{array}{l}2397.6 \\
(880.9)\end{array}$ & -2.63 & 0.008 \\
\hline $\begin{array}{l}\text { E2 on the } \\
\text { day of hCG }\end{array}$ & $\begin{array}{c}3180 \\
(1462.8)\end{array}$ & $\begin{array}{c}3119.2 \\
(1336.8)\end{array}$ & -0.18 & 0.8 \\
\hline $\begin{array}{l}\text { Progesterone } \\
\text { on the day of } \\
\text { hCG }\end{array}$ & $1.2(0.6)$ & $1.2(0.6)$ & -0.459 & 0.6 \\
\hline Total oocte & $25.07(9.2)$ & $18.2(2.9)$ & -7.9 & 0.000 \\
\hline M II oocyte & $19.5(7.2)$ & $14.3(2.9)$ & -7.2 & 0.000 \\
\hline $2 \mathrm{PN}$ & $14.8(6.1)$ & $9.2(2.9)$ & -9.3 & 0.000 \\
\hline
\end{tabular}

As shown in Table 3, in the group that underwent ICSI/ ET with fresh sperm, clinical pregnancy was detected in 25 cases (19.8\%), while no pregnancy was detected in 101 cases $(80.2 \%)$. In the group where ICSI/ET was applied with frozen sperm, clinical pregnancy was detected in 66 cases $(50.8 \%)$, whereas pregnancy was not detected in 64 cases (49.2\%). Clinical pregnancy rates were found to be significantly higher in patients who underwent ICSI/ ET with frozen sperm compared to fresh sperm group ( $50.8 \%$ vs $19.8 \%, \mathrm{p}<.000)$. Similarly, live birth rates were found to be significantly higher in the frozen sperm and ICSI/ET group compared to the fresh sperm group $(3.2 \%$ vs $41.9 \%$, $\mathrm{p}<.00)$.

\begin{tabular}{|lccc|}
\hline Table 3. Chi-square test and Fisher's exact test for both groups \\
\cline { 2 - 3 } Variable & \multicolumn{2}{c|}{ Groups } & \\
& $\begin{array}{c}\text { ICSI with fresh } \\
\text { sperm } \\
\text { N (\%) }\end{array}$ & $\begin{array}{c}\text { ICSI with } \\
\text { frozen sperm } \\
\text { N (\%) }\end{array}$ & P-value \\
\hline $\begin{array}{l}\text { Clinical } \\
\text { pregnancy }\end{array}$ & & & $\mathbf{0 . 0 0 0}$ \\
Yes & $25(19.8)$ & $66(50.8)$ & \\
$\begin{array}{l}\text { No } \\
\text { Live birth }\end{array}$ & $101(80.2)$ & $64(49.2)$ & $\mathbf{0 . 0 0 0}$ \\
Yes & $4(3.2)$ & $54(41.9)$ & \\
No & $122(96.8)$ & $75(58.1)$ & \\
\hline
\end{tabular}

\section{DISCUSSION}

In the present study, we investigated the impact of ICSI with fresh versus cryopreserved testicular sperm on pregnancy outcomes in a large group of patients with azoospermia. The main finding of our study is that clinical pregnancy and live birth rates obtained after ICSI/ET performed with frozen-thawed sperm were found to be significantly higher than ICSI/ET performed with fresh sperm. While our findings are compatible with some studies in the literature, they are incompatible with many studies. Recent meta-analyzes investigating the effects of ICSI/ETs performed with fresh versus frozen sperm on reproductive aoutcome $(4,6)$ reported that there was no difference between the two groups in terms of fertilization, implantation, clinical pregnancy and live birth rates.

However, the results of two comprehensive studies included in the meta-analysis differed significantly from other studies $(4,6)$. In the study conducted by Park et al, although there was no significant difference between the laboratory findings of ICSI cycles using fresh or frozen sperm, implantation rates and pregnancy rates were found to be significantly higher in the frozen-thaw sperm group. However, clinical pregnancy and delivery rates were reported to be similar between the two groups. Our study and the results of Park et al.'s study (5) show many similarities. In our study, both clinical pregnancy rates 
and live birth rates were found to be higher in the frozen sperm group compared to the fresh sperm group. On the other hand, in our study, the total oocyte, M II oocyte and $2 \mathrm{PN}$ embryo counts were found to be significantly higher in the fresh sperm group compared to the frozen sperm group. Although the findings we mentioned last seem paradoxical, they have a scientific explanation. Despite the high number of oocytes and $2 \mathrm{PN}$ embryos in the fresh sperm group, the low pregnancy and delivery rates may be related to the molecular dynamics of early stage fertilization. It is well known fact that during the early stages of fetilization embryo deveopment primarily depend on maternal genome, since the main influence of the paternal transcripts is not occur until the 6-8 cell stage of embryo development (5). For this reason, the reason why CPR and LBR are low in the fresh sperm group despite the better results in the initial laboratory data may be related to the emergence of negative changes due to sperm in the late stage of embryogenesis. As it is known, the age of the expectant mother, the number of total oocytes and embryos, embryo quality and the number of transferred embryos are the main factors affecting pregnancy rates (4-6). The lack of significant difference in demogarfic parameters between the two groups also supported the important role of spermrelated changes in determining fertility prognosis.

The results of the two studies conducted by Madureira et al (7) and $\mathrm{Wu}$ et al (8) differed from both the metaanalysis results and our results. Wu et al (8) demonstrated that the clinical pregnancy rate did not show a significant difference between using fresh or frozen-thawed testicular sperm, but the embryo implantation rates was found significantly different between ICSI with fresh testicular sperm (29.5\%) and ICSI with frozen-thawed testicular sperm(22.2\%) cycles Likewise, Madureira et al (7) reported that comparison between cycles with fresh or frozen-thawed testicular spermatozoa for ICSI led to higher fertilization rate and CPR. When our results, meta-analysis results and other studies are evaluated together, performing ICSI with fresh or frozen testicular sperm does not show a significant effect on clinical pregnancy and live birth rates in most of the studies. In some isolated studies, including our study, an increase was found in both CPR and LBR rates after ICSI with frozen-thawed testicular sperm (4-8).

We do not know the main reason for this difference between studies, but freezing the remaining sperm or testicular tissues after ICSI with fresh sperm in azoospermia patients will save the patient from a repetitive surgical stress and reduce the costs in the next cycle. Having similar or better results in reproductive parameters compared to fresh cycles after ICSI with frozen-thawed sperm is evidence that freezing sperm or testicular tissue is critical for the future fertility of azoospermia patients. During sperm or testis tissue freezing and thawing processes, lipid peroxidation and sperm DNA and acrosomal damage due to free oxygen radicals may occur in the sperm plasma membrane (810). Despite the risk of decrease in sperm count and movement index due to cryoinjury (11), freezing sperm or testicular tissue, if possible, in azoospermia cases, may provide similar or even better clinical pregnancy results than ICSI cycles with fresh sperm.

\section{ETHICAL DECLARATIONS}

Ethics Committee Approval: The study was carried out with the permission of the Research Ethics Committee of Memorial Kayseri Hospital (Date: 16/01/2021, Decision No: 8).

Informed Consent: Verbal and written informed consent was obtained from all participants who participated in this study.

Referee Evaluation Process: Externally peer-reviewed.

Conflict of Interest Statement: The authors have no conflicts of interest to declare.

Financial Disclosure: The authors declared that this study has received no financial support.

Author Contributions: All authors declare that they have all participated in the design, execution, and analysis of the paper and that they have approved the final version.

\section{REFERENCES}

1. Willott GM. Frequency of azoospermia. Forensic Sci Int 1982; 20: 9-10.

2. Franco G, Misuraca L, CilettiM, et al. Surgery of male infertility: an update. RU 2014; 81: 154-64.

3. Ishikawa T. Surgical recovery of sperm in non-obstructive azoospermia. Asian J Androl 2012; 14: 109-15.

4. Yu Z, Wei Z, Yang J, et al. Comparison of intracytoplasmic sperm injection outcome with fresh versus frozen-thawed testicular sperm in men with nonobstructive azoospermia: a systematic review and meta-analysis. J Assist Reprod Genet 2018; 35: 1247 57.

5. Park YS, Lee S-H, Lim CK, Cho JW, Yang KM, Seo JT. Effect of testicular spermatozoa on embryo quality and pregnancy in patients with non-obstructive azoospermia. Syss Biol Reprod Med 2015; 61: 300-6.

6. Ohlander S, Hotaling J, Kirshenbaum E, Niederberger C, Eisenberg ML. Impact of fresh versus cryopreserved testicular sperm upon intracytoplasmic sperm injection pregnancy outcomes in men with azoospermia due to spermatogenic dysfunction: a metaanalysis. Fertil Steril 2014; 101: 344-9.

7. Madureira C, Cunha M, Sousa M, et al. Treatment by testicular sperm extraction and intracytoplasmic sperm injection of 65 azoospermic patients with non-mosaic Klinefelter syndrome with birth of 17 healthy children. Andrology 2014; 2: 623-31.

8. Bin Wu, Wong D, Lu S, Dickstein S, SilvaM, Gelety TJ. Optimal use of fresh and frozen-thawed testicular sperm for intracytoplasmic sperm injection in azoospermic patients. J Assis Reprod Genet 2005; 22: 389-94. 
9. Agca Y, Critser JK. Cryopreservation of spermatozoa in assisted reproduction. Semin Reprod Med. 2002; 20: 15-24

10.Gómez-Torres MJ, Medrano L, Romero A, Fernández-Colom PJ, Aizpurúa J. Effectiveness of human spermatozoa biomarkers as indicators of structural damage during cryopreservation. Cryobiology 2017; 78: 90-4.

11. Chatterjee S, Gagnon C. Production of reactive oxygen species by spermatozoa undergoing cooling, freezing, and thawing. Mol Reprod Dev 2001; 59: 451-8. 